\title{
Estimation of the compounding distribution in the compound Poisson process model for earthquakes
}

\author{
P S MOHARIR \\ National Geophysical Research Institute, Uppal Road, Hyderabad 500007, India \\ MS receivud 6 May 1992; revised 28 September 1992
}

\begin{abstract}
Compound Poisson process models have been studied earlier for earthquake occurrences, with some arbitrary compounding distributions. It is more meaningful to abstract information about the compounding distribution from the empirical observations on the earthquake sequences. The definition of a compound distribution can be interpreted as an integral transform of the compounding distribution. The latter distribution can therefore be estimated by inverting the integral transform. Alternatively, from the moments of the observable random variahles viz. (a) the number of earthquakes per unit time or (b) the waiting times for subsequent earthquakes, the moments of the compounding distribution can be obtained. This information can be converted into a statement about the compounding distribution.
\end{abstract}

Keywords. Compounding distribution; compound Poisson process; waiting time distribution; earthquakes; Hindukush.

\section{Introduction}

\subsection{Poisson process model}

The Poisson process model for earthquake occurrences holds sway due to its appealing simplicity. It can be derived (Fisz 1963) from some simple but ideal assumptions which do not stand the empirical test. Compound Poisson process models (Fisz 1963; Johnson and Kotz 1969, pp 184-196; Naveen et al 1983; Singh 1983) have been proposed as alternatives to the Poisson process model. However, some arbitrary compounding distributions such as gamma, chi and gamma-chi etc. (Naveen et al 1983; Singh 1983) have been used and then the resulting compound process models have been compared against the Poisson process models. A more satisfactory approach would be to derive the compounding distribution from the empirical data about the earthquake occurrences.

To put the ideas more concretely and to set the notation, let $X_{t}$ be the number of earthquakes during the time interval $[0, t)$ where $0 \leqslant t<\infty$. Then $\left\{X_{t}, 0 \leqslant t<\infty\right\}$ constitutes a stochastic process, wherein for every $t$, the labelled random variable $X_{t}$ can take non-negative integer values $k=0,1,2, \ldots$, and as a function of $t, X_{t}$ is a non-decreasing function. If the stochastic process is Poisson

$$
P\left\{X_{t}=k \mid \hat{\lambda}\right\}=\frac{(\hat{\lambda} t)^{k}}{k !} \exp (-\lambda t), k=0,1,2, \ldots ; \lambda>0,
$$

where $P(\cdot)$ is the probability and $\lambda$ is a constant called the rate of the process. Yet 
it is explicitly shown on the left hand side, anticipating further development. One of the consequences of a Poisson process model is obtained by considering a unit interval of time, which, because of the assumption of homogeneity in the derivation of the Poisson process model, (Fisz 1963) can be taken to be $[0,1)$ without loss of generality. Then

$$
P(X=k \mid \lambda)=\lambda^{k} \exp (-\lambda) / k !, k=0,1,2, \ldots, \lambda>0,
$$

where $X$ is the number of earthquakes in unit time interval. Equation (2) defines the Poisson distribution. It is needless to add that it does not necessarily deal with rare phenomena.

Another class of consequences of the Poisson process model relates to the waiting time distributions. Let $T_{q}$ be the waiting time for the $q$ th earthquake, an earthquake having just occurred and let $t_{q}$ be a specific value of the random variable $T_{q}$. Then, under Poisson process model

$$
p_{T}(t \mid \lambda)=\lambda^{q} t^{q-1} \exp (-\lambda t) U(t) /(q-1) !, T=T_{q} ; t=t_{q},
$$

where $p_{Z}(z)$ is the density function of the random variable $Z$ taking values $z$ and $U(\cdot)$ is the unit step function. That is, the waiting time for the qth earthquake is gammadistributed if the earthquakes follow a Poisson process model. In particular, if $q=1$, the waiting time $T_{1}$ for the next earthquake has the density function

$$
P_{T}(t \mid \lambda)=\lambda \exp (-\lambda t) U(t), T=T_{1}, t=t_{1}
$$

which is called the negative exponential density function. $T_{1}$ is the inter-event interval. 'Waiting time' may sound to be an improper term as no one (anxiously) waits for an earthquake, but the term is from a wider mathematical field of queuing theory and a person in a queue does wait to be served. In the present context of earthquakes, it should be taken as a neutral mathematical term.

\subsection{Compound Poisson process model}

It is generally agreed that the successive inter-event arrival times are dependent on the sequence of earthquake magnitudes. That is, one must generalize to "marked" Poisson process models, magnitude being one of the "marker" variables for the earthquake events. As a half-way measure, here it is only assumed that the earthquakes cannot be adequately characterized as drawn from a process with a constant rate $\lambda$. Therefore, it is awarded the status of a random variable, whose actual values are determined by the sequence of earthquake magnitudes. The density function $p(\lambda)$ is assumed to summarize that inadequately modelled aspect.

From equation (1), it follows that

$$
P\left(X_{t}=k\right)=\int_{0}^{\infty} \frac{(\lambda t)^{k}}{k !} \exp (-\lambda t) p(\lambda) \mathrm{d} \lambda, k=0,1,2, \ldots
$$

Correspondingly (2) to (4) become

$$
P(k)=P(X=k)=\frac{\lambda^{k}}{k !} \exp (-\lambda) p(\lambda) \mathrm{d} \lambda, k=0,1,2, \ldots
$$




$$
p_{T}(t)=\int_{0}^{\infty} \frac{\lambda^{q} t^{q-1}}{(q-1) !} \exp (-\lambda t) p(\lambda) \mathrm{d} \lambda, T=T_{q}, t=t_{q}>0
$$

and

$$
p_{T}(t)=\int_{0}^{\infty} \lambda \exp (-\lambda t) p(\lambda) \mathrm{d} \lambda, T=T_{1}, t=t_{1}>0
$$

for the compound (a) Poisson process, (b) Poisson distribution, (c) gamma, and (d) negative exponential density functions, respectively. The density function $p(\lambda)$ of $\lambda$ is then called the compounding density function.

The problem posed here is: if observations on the random variables $X$ and $T_{q}$ are available, how could $p(\lambda)$ be estimated?

\section{Integral transform approach}

It needs only a slight reorientation to recognize (6) to (8) as integral transforms of $p(\lambda)$, yielding $P(k), p_{T_{q}}\left(t_{q}\right)$ and $p_{T_{1}}\left(t_{1}\right)$ as transforms (Walter 1985). Therefore, one can directly ask whether these transforms can be inverted. Further, it would be advantageous if some rearrangement would bring out the well-known integral transforms.

Equation (7) can be rearranged as

$$
(q-1) ! t^{1-q} p_{T}(t)=\int_{0}^{\infty} p(\lambda) \lambda^{q} \exp (-\lambda t) \mathrm{d} \lambda, T=T_{q}, t=t_{q}
$$

If the variable $\lambda$ is replaced by $x$ and $t_{q}$ by $y,(9)$ is of the form

$$
F(y)=\int_{0}^{\infty} f(x) \exp (-x y) \mathrm{d} x
$$

where

and

$$
F(y)=(q-1) ! y^{1-q} p_{T}(y), T=T_{q}
$$

$$
f(x)=p(x) x^{q}
$$

From (10), it follows that $f(x)$ and $F(y)$ constitute a Laplace transform pair. Therefore, $f(x)$ can be obtained as the inverse Laplace transform of $F(y)$. This gives a formal solution to the problem of estimating the compounding distribution $p(\lambda)$ given $p_{T_{q}}\left(t_{q}\right)$. From the latter, which will have to be estimated from the knowledge of the origin times of a sequence of earthquakes, the Laplace transform $F(y)$ can be computed. Inverse Laplace transform then gives $f(x)$, from which $p(\lambda)$ can be obtained. The solution has been called formal, because there are difficulties. In the $y$ domain, $F(y)$ has a singularity at origin, unless $q=1$, and division by $x^{q}$ after inverse Laplace transformation also will create a difficulty at the origin. Numerical inversion of Laplace transform (Bellman et al 1966) is also a difficult proposition, Laplace transform being more a theoretical device to get powerful results, rather than a numerical tool. Further, estimation of the density function (Goel et al 1983; Moharir 1992a) of $T_{q}$ from its samples is not a straightforward problem either. Inversion of $(8)$ for $p(\lambda)$ is a special case of the procedure above, corresponding to $q=1$. 
Equation (6) can be written as

$$
k ! P(k)=\int_{0}^{\infty}\{p(\lambda) \exp (-\lambda) / \lambda\} \lambda^{k-1} \mathrm{~d}(\lambda) .
$$

Again, if with change of variable $p(\lambda) \exp (\lambda) / \lambda$ is written as $f(x)$ and $k ! P(k)$ as $F(y)$, then (13) becomes

$$
F(y)=\int_{0}^{\infty} f(x) x^{y-1} \mathrm{~d} x,
$$

so that $f(x)$ and $F(y)$ can now be seen as a Mellin transform pair (Sneddon 1972) with a difference that in Mellin transform, $y$ is a continuous variable, whereas $k$ in the present context is a non-negative integer. As the intended inverse Mellin transform would have to be performed numerically and as it would involve such a discretization, this is not a grave difficulty. So, from the estimates of the probabilities $P(k), F(y)$ could be derived and $f(x)$ obtained from it by numerical inverse Mellin transformation, from which, $p(\lambda)$ can, in principle, be obtained. The estimation of probability of a discrete random variable is not again a simple problem. It has answers (Johnson and Kotz 1969, pp 94-95) for Poisson distribution, but they may not carry over readily for compound Poisson distribution with unknown compounding distribution. Numerical inversion of Mellin transform could provide its own headaches and multiplication by $k$ ! and $\lambda \exp (\lambda)$ may pose problems. Herce, this solution also should be regarded only as formal.

\section{Estimation of the moments of the compounding distribution}

In this section, the moments of the observable random variables, viz. (a) the number of earthquakes per unit time and (b) the waiting times for future earthquakes, are related to the moments of the compounding density function $p(\lambda)$.

\subsection{A case of compound Poisson distribution}

The $n$th moment of the compounding density function $p(\lambda)$ is denoted by $\mu_{n}$ and the $n$th moment of the number of earthquakes per unit time interval by $\mu_{n}^{\prime}$. From (6)

$$
\begin{aligned}
\mu_{n}^{\prime} & =E\left(X^{n}\right)=E\left(k^{n}\right)=\sum_{k=0}^{\infty} k^{n} P(k) \\
& =\sum_{k=0}^{\infty} k^{n}(k !)^{-1} \int_{0}^{\infty} \lambda^{k} \exp (-\lambda) p(\lambda) \mathrm{d} \lambda \\
& =\sum_{k=0}^{\infty} k^{n}(k !)^{-1} \sum_{m=0}^{\infty}(-1)^{m}(m !)^{-1} \mu_{k+m^{\prime}}
\end{aligned}
$$

where $E(\cdot)$ denotes the 'expected value of'. Equation (15) indicates that the moments of the number of earthquakes per unit time interval can be expressed in terms of the 
moments of the compounding density function $p(\lambda)$ (Tucker 1963). To express the latter in terms of the former, which are empirically estimable, the relations of (15) have to be inverted. Towards that end, (15) can be simplified by putting

$$
k+m=r
$$

to get

$$
\mu_{n}^{\prime}=\sum_{r=1}^{\infty} \mu_{r} \sum_{m=0}^{r-1}(-1)^{m}(m !)^{-1}(r-m)^{n-1}((r-m-1) !)^{-1}
$$

It can be shown that

$$
\sum_{m=0}^{r-1}(-1)^{m}(m !)^{-1}(r-m)^{n-1}((r-m-1) !)^{-1}=0, r=n+1, n+2, \ldots
$$

Therefore (17) further reduces to

$$
\mu_{n}^{\prime}=\sum_{r=1}^{n} \mu_{r} \sum_{m=0}^{r-1}(-1)^{m}(m !)^{-1}(r-m)^{n-1}((r-m-1) !)^{-1}
$$

Thus, the nth moment of $X$ (or $k$ ) depends only on the moments up to $n$ th, of the compounding density function. This could have been anticipated. Equation (19) can be solved to yield

$$
\begin{aligned}
& \mu_{1}=\mu_{1}^{\prime}, \\
& \mu_{2}=-\mu_{1}^{\prime}+\mu_{2}^{\prime}, \\
& \mu_{3}=2 \mu_{1}^{\prime}-3 \mu_{2}^{\prime}+\mu_{3}^{\prime}, \\
& \mu_{4}=-6 \mu_{1}^{\prime}+11 \mu_{2}^{\prime}-6 \mu_{3}^{\prime}+\mu_{4}^{\prime}, \\
& \mu_{5}=24 \mu_{1}^{\prime}-50 \mu_{2}^{\prime}+35 \mu_{3}^{\prime}-10 \mu_{4}^{\prime}+\mu_{5}^{\prime}, \\
& \mu_{6}=-120 \mu_{1}^{\prime}+274 \mu_{2}^{\prime}-225 \mu_{3}^{\prime}+85 \mu_{4}^{\prime}-15 \mu_{5}^{\prime}+\mu_{6}^{\prime}, \\
& \mu_{7}=720 \mu_{1}^{\prime}-1764 \mu_{2}^{\prime}+1624 \mu_{3}^{\prime}-735 \mu_{4}^{\prime}+175 \mu_{5}^{\prime}-21 \mu_{6}^{\prime}+\mu_{7}^{\prime}, \ldots
\end{aligned}
$$

It turns out (Walter 1985) that there is a simple pattern in (20). The $n$th factorial moment (Johnson and Kotz 1969, p. 19) of $X$ (or $k$ ) is defined as

$$
\mu_{f n}^{\prime}=E[k(k-1) \ldots(k-n+1)]
$$

and is a linear combination of $\mu_{1}^{\prime}, \mu_{2}^{\prime}, \ldots, \mu_{n}^{\prime}$. These linear combinations are precisely those in (20), so that it can be summarized as

$$
\mu_{n}=\mu_{f n}^{\prime}, n=1,2, \ldots
$$

That is, the $n$th moment of the compounding density function $p(\lambda)$ is the $n$th factorial moment of the number of earthquakes per unit time interval. Equation (22) strikes as a simple relation, (20) does not. Yet, they are equivalent. Simplicity, thus, is a matter of expression. 
3.2 A case of waiting time distributions

Using (7)

$$
\begin{aligned}
\tilde{\mu}_{n, q} & =E\left[t^{n}\right]=\int_{0}^{\infty} t^{n} p_{T}(t) \mathrm{d} t \\
& =\int_{0}^{\infty} \mathrm{d} t t^{n} \int_{0}^{\infty} \mathrm{d} \lambda p(\lambda) \lambda^{q} t^{q-1} \exp (-\lambda t)((q-1) !)^{-1} \\
& =\int_{0}^{\infty} \mathrm{d} \lambda p(\lambda) \lambda^{q}((q-1) !)^{-1} \int_{0}^{\infty} \mathrm{d} t t^{n+q-1} \exp (-\lambda t), \\
T & =T_{q}, t=t_{q}
\end{aligned}
$$

where $\tilde{\mu}_{n, q}$ is the $n$th moment of the waiting time for the $q$ th earthquake. Using

$$
\int_{0}^{\infty} t^{n-1} \exp (-\lambda t) \mathrm{d} t=\Gamma(n) \lambda^{-n}
$$

Equation (23) gives

$$
\begin{aligned}
\tilde{\mu}_{n, q} & =\int_{0}^{\infty} \mathrm{d} \lambda p(\lambda) \lambda^{-q} \Gamma(n+q)((q-1) !)^{-1} \lambda^{-(n+q)} \\
& =\left[\Gamma(n+q)((q-1) !)^{-1}\right] \int_{0}^{\infty} \lambda^{-n} p(\lambda) \mathrm{d} \lambda \\
& =\Gamma(n+q)((q-1) !)^{-1} \mu_{-n} .
\end{aligned}
$$

In the derivation of (25), there is no restriction on $n$. Habitually, it is assumed to be a positive integer, in which case $\mu_{-n}$ could be called the $n$th inverse moment (Johnson and Kotz 1969, pp 52-53) of the compounding density function. For $q=1$, (25) simplifies to

$$
\tilde{\mu}_{n, 1}=\Gamma(n+1) \mu_{-n}=n ! \mu_{-n},
$$

which is as simple a relation as that of (22).

Equations (25) and (26) can be combined to yield

$$
\tilde{\mu}_{n, q} / \tilde{\mu}_{n, 1}=\left(\begin{array}{c}
n+q-1 \\
n
\end{array}\right)=\left(\begin{array}{c}
n+q-1 \\
q-1
\end{array}\right)
$$

in which the $\mu_{-n}$ term, or in other words, the compounding density function, is eliminated. Equation (27) prescribes how the moments of the various waiting time distributions should be related under the assumption of a compound Poisson process model. The same relations hold even for the Poisson process model, as can be readily verified. This is not surprising because the Poisson process model is a particular case of a compound Poisson model. Indeed, any relation for the latter, not depending on the compounding density function, must also hold for the Poisson process. The implication of this observation is that comparison of the moments of various waiting 
time distributions in the context of (27) sheds no light on the appropriateness of a compound Poisson process model over the Poisson process model. Alternatively, if on the basis of such a comparison, the Poisson process model for earthquake occurrences is rejected, all the compound Poisson process models also will be of no avail. This makes compound Poisson process models attractive or unacceptable depending on whether (27) is adjudged as satisfied or not.

Equation (25) can be written as

$$
\mu_{-n, q}=((q-1) ! / \Gamma(n+q)) \tilde{\mu}_{n, q}
$$

wherein the notation is slightly more elaborate. Simple $\mu_{-n}$ for the $n$th inverse moment is replaced by $\mu_{-n, q}$, the additional subscript being a reminder that the estimate of $\mu_{-n}$ is obtained using the moments of the $q$ th waiting time distribution. It is actually not necessary that $n$ be regarded as a positive integer. Therefore, changing the sign of $n$

$$
\mu_{n, q}=((q-1) ! / \Gamma(q-n)) \tilde{\mu}_{-n, q}
$$

If $n$ in (29) is regarded to be a positive integer, the argument of the gamma function should be kept positive to avoid its irregular behaviour for negative integer values. This leads to the restriction that

$$
q=n+1, n+2, \ldots
$$

That is, $\mu_{1}$ can be estimated only from the waiting times for the second earthquake onwards, $\mu_{2}$ cannot be estimated without going to waiting times for the third earthquake onwards, etc. This, in retrospect, is the reason why the analysis is being conducted for the waiting times of all the future earthquakes, even though inter-event interval is the only basic concept from which other waiting times can be derived.

\section{The use of these results}

The moments, (conventional, factorial and inverse) of the observables of the earthquake processes, such as the number of earthquakes per unit time interval and the waiting times for later earthquakes, can be related to the moments (conventional and inverse) of the compounding distributions. Once the latter are known, any method of estimating the density function, given the moments can be used. One conventional method is based on the Gram-Charlier series truncated according to the Edgeworth pattern (Fry 1965). It is not appropriate for the variables like $\lambda$, defined over the interval $[0, \infty)$. Hence Laguerre type Gram-Charlier method was developed (Goel et al 1983). Its merit has also been recognized by others (Waiter 1985). However, Gram-Charlier methods adopted for any range of definition, still have the disadvantage of employing the notion of orthonormality, which is appropriate for the $L^{(2)}$ class of functions, but not for density functions, which are forbidden to take negative values. Therefore, a density function $p(\lambda)$ is written below as a sum of standard density functions, such as the gamma. It is shown that it leads to a nonlinear mathematical programming problem, if the condition that $\beta_{i} \geqslant 0$ (see next section) is to be imposed. If it is not imposed as a part of the solution procedure, nonacceptable solutions may result. 


\section{Application to field data}

The data of earthquakes in the Hindukush region are used. They are taken from the catalogue published by the India Meteorological Department. For computing the number of earthquakes per unit time, 2395 earthquakes of magnitude greater than 3.5 for the period from January 1963 to December 1974, having focal depth less than $250 \mathrm{~km}$ in an area bounded by $62^{\circ}$ to $76^{\circ} \mathrm{E}$ longitude and 30 to $39^{\circ} \mathrm{N}$ latitude are used.

Using one week as a unit of time, the moments of the random variable $\mathrm{X}$, i.e. the number of earthquakes per week, are (Naveen et al 1983)

$$
\mu_{1}^{\prime}=3 \cdot 281, \mu_{2}^{\prime}=17 \cdot 287, \mu_{3}^{\prime}=113 \cdot 64 \text {. }
$$

Using (20), the moments of $\lambda$ are

$$
\mu_{1}=3 \cdot 281, \mu_{2}=14 \cdot 006, \mu_{3}=68 \cdot 34 \text {. }
$$

One way of constructing the density function $p(\lambda)$ from the moments is to assume

$$
p(\lambda)=\sum_{i=1}^{r} \beta_{i} p_{i}(\lambda)
$$

where $p_{i}(\lambda)$ are standard density functions and $\beta_{i}$ are positive constants that add up to unity.

Then it readily follows that

$$
\mu_{n}=\sum_{i=1}^{r} \beta_{i} \mu_{n}^{(i)}
$$

where $\mu_{n}^{(i)}$ is the $n$th moment of $p_{i}(\lambda)$.

If $p_{i}(\lambda)$ are chosen to be Pascal density functions (gamma with $q$ in (3) to be positive integers), i.e. if

then

$$
p_{i}(\lambda)=\alpha_{i}^{i} \lambda^{i-1}((i-1) !)^{-1} \exp \left(-\alpha_{i} \lambda\right) U(\lambda)
$$

$$
\mu_{n}^{(i)}=i(i+1)(i+2) \ldots(n+i-1) \alpha_{i}^{-n},
$$

where $\alpha_{i} s$ are constants. Thus, in general, (34) is not a linear set of equations, with $\beta_{i} s$ as the only constants to be evaluated. Because, $\mu_{n}^{(i)} s$ involve the parameters of the standard density functions $p_{i}(\lambda)$, and they too would have to be evaluated.

In particular, one could choose

$$
\mu_{1}=1 / \alpha_{1}=2 / \alpha_{2}=3 / \alpha_{3}=\ldots
$$

implying that all the standard density functions $p_{i}(\lambda)$ of $(35)$ are chosen to have the same mean. Then (34) leads to

$$
\left[\begin{array}{c}
1 \\
\mu_{2} / \mu_{1}^{2} \\
\mu_{3} / \mu_{1}^{3} \\
\mu_{4} / \mu_{1}^{4} \\
\cdot \\
\cdot
\end{array}\right]=\left[\begin{array}{cccc}
1 & 1 & 1 & 1 \\
2 & 3 / 2 & 4 / 3 & 5 / 4 \\
6 & 3 & 20 / 9 & 15 / 8 \\
24 & 12 / 2 & 40 / 9 & 105 / 32 \\
\cdot & \cdot & \cdot & \cdot \\
\cdot & \cdot & \cdot & \cdot \\
\cdot & \cdot & \cdot & \cdot
\end{array}\right]\left[\begin{array}{c}
\beta_{1} \\
\beta_{2} \\
\beta_{3} \\
\beta_{4} \\
\cdot \\
\cdot \\
\cdot
\end{array}\right]
$$


The condition that the $\beta_{l} s$ should add up to unity is included in (38) automatically, but the condition that the $\beta_{t} s$ should be positive is not. The latter condition makes the problem that of mathematical programming.

Using estimates of (32), (38) gives, after truncation at $r=3$,

$$
\left[\begin{array}{l}
1 \\
1 \cdot 301 \\
1.935
\end{array}\right]=\left[\begin{array}{ccc}
1 & 1 & 1 \\
2 & 3 / 2 & 4 / 3 \\
6 & 3 & 20 / 9
\end{array}\right]\left[\begin{array}{l}
\beta_{1} \\
\beta_{2} \\
\beta_{3}
\end{array}\right]
$$

This does not yield an acceptable solution for $\beta_{i} s$. Truncation at $r=2$ does not help either.

Using the gamma density functions, rather than Pascal as standard density functions gives

$$
p_{i}(\lambda)=a_{i}^{v_{i}} \lambda^{v_{i}-1} \exp \left(a_{i} \lambda\right) U(\lambda) / \Gamma\left(v_{i}\right)
$$

so that each standard density function has two parameters $a_{i}$ and $v_{i}$. If $r$ of them are used, there would be $(r-1)$ values of $\beta_{i} s$ to be determined (because $r$ of them must add up to unity). Thus there would be in all $(3 r-1)$ parameters to be estimated, requiring at least $(3 r-1)$ moments of $p(\lambda)$ to be used. Taking the simplest case of $r=1$ and using the first two moments of (32), we get

$$
a=1 \cdot 012, \quad v=3 \cdot 321,
$$

where subscripts of $a$ and $v$ in (40) are dropped, as only one value of $i$ is used. This is obviously the same result as was obtained earlier (Naveen et al 1983) when gamma distribution was used to be the compounding distribution.

From the waiting time values it was found that ( $\tilde{\mu}_{n}$ values in seconds)

and

$$
\begin{array}{ll}
\tilde{\mu}_{1,1}=1.664 \times 10^{5}, & \tilde{\mu}_{2,1}=6.473 \times 10^{10}, \\
\tilde{\mu}_{3,1}=4.341 \times 10^{16}, & \tilde{\mu}_{4,1}=4.023 \times 10^{22}, \\
\tilde{\mu}_{1,2}=3.375 \times 10^{5}, & \tilde{\mu}_{2,2}=1.821 \times 10^{11}, \\
\tilde{\mu}_{3,2}=1.415 \times 10^{17}, & \tilde{\mu}_{4,2}=1.473 \times 10^{23},
\end{array}
$$

$$
\begin{aligned}
& \tilde{\mu}_{1,3}=5.037 \times 10^{5}, \quad \tilde{\mu}_{2,3}=3.443 \times 10^{11}, \\
& \tilde{\mu}_{3,3}=2.972 \times 10^{17}, \quad \tilde{\mu}_{4,3}=3.095 \times 10^{23},
\end{aligned}
$$

Then (28) yields

and

$$
\begin{array}{ll}
\mu_{-1,1}=1.664 \times 10^{5}, & \mu_{-2,1}=3.236 \times 10^{10} \\
\mu_{-3,1}=7.235 \times 10^{15}, & \mu_{-4,1}=1.676 \times 10^{21} \\
\mu_{-1,2}=1.687 \times 10^{5}, & \mu_{-2,2}=3.035 \times 10^{10} \\
\mu_{-3,2}=5.894 \times 10^{15}, & \mu_{-4,2}=1.227 \times 10^{21}
\end{array}
$$

$$
\begin{array}{ll}
\mu_{-1,3}=1.679 \times 10^{5}, & \mu_{-2,3}=2.869 \times 10^{10}, \\
\mu_{-3,3}=4.954 \times 10^{15}, & \mu_{-4,3}=8.597 \times 10^{20} .
\end{array}
$$


From (42)-(44)

and

$$
\begin{array}{ll}
\tilde{\mu}_{1,2} / \tilde{\mu}_{1,1}=2 \cdot 028(2), & \tilde{\mu}_{2,2} / \tilde{\mu}_{2,1}=2 \cdot 813(3), \\
\tilde{\mu}_{3,2} / \tilde{\mu}_{3,1}=3 \cdot 259(4), & \tilde{\mu}_{4,2} / \tilde{\mu}_{4,1}=3.661(5),
\end{array}
$$

$$
\begin{array}{ll}
\tilde{\mu}_{1,3} / \tilde{\mu}_{1,1}=3.026(3), & \tilde{\mu}_{2,3} / \tilde{\mu}_{2,1}=5.319(6), \\
\tilde{\mu}_{3,3} / \tilde{\mu}_{3,1}=6.847(10), & \tilde{\mu}_{4,3} / \tilde{\mu}_{4,1}=7.693(15),
\end{array}
$$

where the theoretical values of these ratios under the assumption of compound Poisson process models (including the Poisson process model as a special case), obtained from (27), are shown in parentheses, for comparison. The deviation from the theoretical expectation is greater for higher moments and farther future earthquakes. It reflects in the differences among various estimates of the inverse moments of the presumed compounding distribution also.

Equation (34) can equally well be written as

$$
\mu_{-n}=\sum_{i=1}^{r} \beta_{i} \mu_{-n}^{(i)}
$$

If the standard density functions are Pascal of (35), then

$$
\mu_{-n}^{(i)}=(i-n-1) ! /\left[(i-1) ! \alpha_{i}^{m}\right] .
$$

For these inverse moments to exist, it is necessary that $n \leqslant i-1$. That is, no inverse moments exist for $p_{1}(\lambda)$ of (35), i.e. for the negative exponential density function, only the first inverse moment exists for $p_{2}(\lambda)$, etc. That is, density functions of (35) are not the proper density functions in terms of which to construct the compounding distribution of the Poisson process model, from its inverse moments.

Alternatively, the conventional moments of the compounding distribution can be obtained by using (29), with the restriction of (30), that for estimating higher moments of the compounding distribution, the waiting times of only later earthquakes can be used. Following that restriction

$$
\begin{aligned}
& \mu_{1,2}=5.272 \times 10^{-6}, \quad \mu_{1,3}=6.673 \times 10^{-6} \\
& \mu_{2,3}=7.027 \times 10^{-12}
\end{aligned}
$$

Using $\mu_{1,3}$ and $\mu_{2,3}$ as the estimates of $\mu_{1}$ and $\mu_{2}$, respectively and using (38), truncated for $r=2$ yields

$$
\left[\begin{array}{l}
1 \\
1 \cdot 578
\end{array}\right]=\left[\begin{array}{ll}
1 & 1 \\
2 & 3 / 2
\end{array}\right]\left[\begin{array}{l}
\beta_{1} \\
\beta_{2}
\end{array}\right]
$$

which gives

$$
\beta_{1}=0 \cdot 156, \quad \beta_{2}=0 \cdot 844 \text {. }
$$

However, instead of $\mu_{1,3}$ if $\mu_{1,2}$ is used as the estimate of $\mu_{1}$ above, an unacceptable solution is obtained.

It thus seems desirable to construct the compounding distribution in terms of density functions for which the conventional as well as inverse moments exist. One 
simple choice would be a uniform density function over the range $\lambda_{m}$ to $\lambda_{M}$. These two parameters can be estimated from $\mu_{-1}$ and $\mu_{1}$. It readily follows that

$$
\begin{aligned}
\mu_{-1} & =\left(\lambda_{M}-\lambda_{m}\right)^{-1} \ln \left(\lambda_{M} / \lambda_{m}\right), \\
\mu_{1} & =\left(\lambda_{M}+\lambda_{m}\right) / 2
\end{aligned}
$$

Therefore

$$
\lambda_{M}=2 \mu_{1}-\lambda_{m}
$$

where $\lambda_{m}$ is obtained by satisfying

$$
\mu_{-1}=\left[2\left(\mu_{1}-\lambda_{m}\right)\right]^{-1} \ln \left[\left(2 \mu_{1}-\lambda_{m}\right) / \lambda_{m}\right] \text {. }
$$

Using $\mu_{1,3}$ of (52) and $\mu_{-1,3}$ of (47) as the estimates of $\mu_{1}$ and $\mu_{-1}$, respectively (both obtained from the waiting times of the third earthquake) yields

$$
\lambda_{m}=3.05 \times 10^{-6}, \quad \lambda_{M}=10.3 \times 10^{-6} .
$$

It may also be of interest to note that one readily falls prey to the unaccountable influence of countable and ordinal numbers. Rather than recognizing (40) as a doubly infinite family of density functions, one may unwittingly substitute $v_{i}=i$ to get a family of one-parameter Pascal density functions, the other parameter being frozen to take only integer values. Further, one may equally unwittingly truncate their contributions by including only the first (lowest) $r$ values of $i$. These decisions may not have any justification beyond habit. Similarly one mechanically thinks in terms of $n$th moments, $n$ a positive integer. The treatment here has naturally led us to inverse moments, so that $n=-1,-2, \ldots$ But there is no need to restrict $n$ to integer values. For example, from the inter-event interval data it is possible to obtain

$$
\tilde{\mu}_{-1 / 2,1}=4.765 \times 10^{-3}
$$

and hence from (29)

$$
\mu_{1 / 2,1}=8.446 \times 10^{-3}
$$

Relations such as (38) based on integer moments may be ill-conditioned. Note the serious lack of diagonal dominance in equation (38), as an indication. By using fractional moments such as in (60), the ill-conditioning could be significantly constrained.

\section{Recapitulation and discussion}

Compound Poisson process and distributions derived from it can be viewed as integral transforms of the compounding distribution. This may lead to methods of estimating the compounding distribution, provided the associated difficulties are sorted out. That problem has not been attempted here.

It is demonstrated that it is useful to write $p(\lambda)$ as a convex combination of standard density functions for which the conventional and inverse moments both exist. Gamma density functions do not satisfy this condition. Beta density functions defined over a 
finite support may be a good choice. Simple uniform distribution may also be a good choice for a compounding distribution.

It is demonstrated that different derivations from the compound Poisson process model, such as the compound Poisson distribution for the number of earthquakes per unit time interval and gamma distributions for the waiting times for different future earthquakes, lead to different estimates for the compounding distribution. This takes various forms such as not all derivations being amenable to such an estimation, some derivations being amenable to the estimation of the compounding distribution by only particular methods or in estimates being different. This can be understood readily. Compound Poisson process is merely a model. The actual earthquake sequence is a "marked" stochastic process and that aspect is ignored here, though the marker variables have a bearing on the observables considered here. Secondly, the actual stochastic process may not be Poisson. The deviations from the model may afterall be differently projected onto different derivations from the model. In other words, the estimates of the compounding distribution from different derivations from the compound Poisson process model can have different sensitivities to the deviations from that model. This is fertile ground for "equivocal equivalences", "proximity reversals" and other attendant paradoxes (Moharir 1992(b), 1993), which are very relevant, indeed, but not discussed here, as they would need a fresh introduction. The problems are not eliminated by increasing the number of moments included in the estimation procedure.

The limitation of the compound Poisson process models as alternatives to the Poisson process model has been brought out. The relations between the moments of various waiting time distributions are not affected by compounding. If these relations are not satisfied by the empirical data, one may have to look beyond compounding for proper explanation.

\section{Acknowledgements}

The author is thankful to Dr H N Srivastava, Meteorological Office, Pune for giving the Hindukush data. He is grateful to some members of the Research Council of NGRI for being instrumental in prompting and requiring him to take interest in this field. Thanks are also due to Prof D Guptasarma for providing leisure, and a good environment to work in. The author is grateful to the referees.

\section{References}

Bellman R, Kalaba R E and Lockett J A 1966 Numerical Inversion of the Laplace transform (New York: American Elsevier) pp 249

Fisz M 1963 Probability theory and mathematical statistics (New York: John Wiley) pp 52-85

Fry T C 1965 Probability and its engineering uses (Princeton: D. Von Nostrand) pp 257-270

Goel S M J R, Malasi S K, Moharir P S, Wason H R, Khattri K N and Gaur V K 1983 Estimation of the waiting time distributions of earthquakes; Proc. Indian Acad. Sci. (Earth Planet. Sci.) 92 223-237

Johnson N L and Kotz S 1969 Discrete distributions (Boston: Houghton Miflin Company) pp 328

Moharir P S 1992(a) Pattern-recognition transforms (Taunton: Research Studies Press) pp 30-38

Moharir P S 1992(b) Themata and anathemata in signal processing. Geol. Soc. India Mem. 24 (in press)

Moharir P S 1993 Induced condorcet paradox. Proc. Discussion Meeting on Signal Processing \& Communications, Bangalore 
Naveen, Moharir P S and Gaur V K 1983 Hypotheses for earthquake occurrences; Proc. Indian Acad. Sci. (Earth Planet. Sci.) 92 261-281

Singh H 1983 Compound Poisson process models for earthquake occurrences. M. Tech. Dissertation, University of Roorkee, Roorkee

Sneddon I N 1972 The use of integral transforms (New York: McGraw-Hill)

Tucker H G 1963 An estimate of the compounding distribution of a compound Poisson distribution; Theory Probab. Appl. 8 195-200

Walter C G 1985 Orthogonal polynomial estimators of the prior distribution of a compound Poisson distribution. Sankhya (Indian J. Stat.) A47 222-230 